\section{Medical Principles} and Practice

\section{Renu Gupta ${ }^{\mathrm{a}}$ \\ Manpreet Gambhir ${ }^{\mathrm{b}}$ \\ A.C. Ammini}

a Department of Radiology, Faculty of Medicine, Kuwait University,

Kuwait;

Departments of

${ }^{b}$ Radiology and

c Endocrinology, All-India Institute of Medical Sciences, New Delhi, India

\title{
Imaging of the Pituitary Gland in Cushing's Disease
}

\section{Key Words}

Computed tomography

Magnetic resonance imaging

Pituitary gland

Cushing's disease

\begin{abstract}
Objective: To evaluate the sensitivity of computed tomography (CT) and magnetic resonance imaging (MRI) of the pituitary gland in detecting pituitary adenomas in patients with Cushing's disease. Methods: Twenty-five patients aged 8-55 years (average age 30 years), clinically and biochemically diagnosed as having Cushing's disease, were evaluated with a contrast-enhanced CT scan and MRI of the pituitary gland. Contrast-enhanced CT scans of direct 2-mm-thick coronal sections were performed. Pre- and post-enhanced MRI of the pituitary gland was performed on a 1.5-tesla superconducting magnet, taking 2-mm-thick sections in the sagittal and coronal planes. Results: MRI revealed adenomas in 16 patients (12 microadenomas and 4 macroadenomas). Twelve (75\%) adenomas were delineated more clearly on post-gadolinium MRI scans. Nine $(56 \%)$ adenomas were better delineated on coronal images. Out of 16 adenomas identified on the MRI, only 5 were seen on the CT scan. All patients underwent trans-sphenoid surgery and the MRI findings were confirmed. Conclusion: Contrast-enhanced MRI is the preferred modality for the detection of ACTH-secreting adenomas, which are difficult to visualize on CT scans due to their small size. A focal hypointense lesion is the commonest finding on $\mathrm{T}_{1}$-weighted images and, if identified on coronal sections, is the most sensitive diagnostic criterion. However, a negative MRI does not exclude the presence of a microadenoma.
\end{abstract}

\begin{tabular}{ll}
\hline KARGER & ○ 1998 S. Karger AG, Basel \\
Fax +41 61 306 12 34 & 1011-7571/98/0074-0246\$15.00/0 \\
$\begin{array}{l}\text { E-Mail karger@karger.ch } \\
\text { www.karger.com }\end{array}$ & $\begin{array}{l}\text { Accessible online at: } \\
\text { http://BioMedNet.com/karger }\end{array}$
\end{tabular}

Dr. Renu Gupta

Department of Radiology, Faculty of Medicine, Kuwait University PO Box 24923, 13110 Safat (Kuwait) Tel. +965 53304 73, Fax +9655338936 E-Mail Renu@hscc.kuniv.edu.kw 


\section{Introduction}

Cushing's disease is characterized by pituitary hypersecretion of ACTH leading to a gamut of clinical features of chronic hypercortisolism including obesity, hypertension, hirsutism, cutaneous striae, menstrual irregularity, acne and fatigue. A pituitary adenoma as the cause of ACTH hypersecretion is seen in $60-90 \%$ of the patients. Trans-sphenoid microsurgical techniqe has made the accurate pre-operative localization of pituitary adenomas mandatory. Selective removal of adenomas not only cures the symptoms arising from ACTH hypersecretion, but also prevents postoperative pituitary insufficiency that might arise from extensive pituitary exploration.

Pituitary adenomas represent only 50\% of adenomas detected on computed tomography (CT) scans. Magnetic resonance imaging (MRI) significantly improves the ability to detect microadenomas in Cushing's disease. MRI, especially after injection of intravenous gadolinium-DTPA, has a reported sensitivity of $71 \%$ and a specificity of $87 \%$ [1] in detecting pituitary adenomas. As a result, it has become the imaging technique of choice for pre-operative evaluation of Cushing's disease.

\section{Methods}

Twenty-five patients with symptoms of hypercortisolism were evaluated pre-operatively with contrastenhanced CT and MRI of the pituitary. The biochemical diagnosis of hypercortisolism was made when basal levels were high, diurnal variation was lost and/or the cortisol level was not reduced after oral administration of low-dose dexamethasone $(0.5 \mathrm{mg}$ every $6 \mathrm{~h}$ for 2 days).

All patients were first examined by CT. Two-millimetre-thick direct coronal slices of the sella were obtained on a third-generation CT scanner (Somatom DR, Siemens, Germany) after intravenous injection of 40-60 $\mathrm{ml}$ of an iodinated contrast medium (Urografin $76 \%$, Schering AG, Germany).
Thereafter, MRI of the pituitary was performed on a 1.5-tesla superconducting MR system (Magentom SP 4000, Siemens, Germany). Serial multisection $\mathrm{T}_{1^{-}}$ weighted images (TR/TE 450/15) were obtained in the sagittal and coronal planes using a circularly polarized head coil. All examinations were performed using a $20-\mathrm{cm}$ FOV matrix of $256 \times 256,2$ excitations and slices of $2 \mathrm{~mm}$ thickness. Post-contrast images were obtained after intravenous injection of gadoliniumDTPA at a dose of $0.1 \mathrm{mmol} / \mathrm{kg}$ body weight. An experienced radiologist evaluated the images, and each case was discussed with a neuroradiologist.

All the patients with pituitary adenomas subsequently underwent trans-sphenoidal pituitary microsurgery for the removal of the adenomas. When pituitary surgery could not be performed for technical reasons, or when pituitary lesions could not be identified, the patients were subjected to bilateral adrenectomy.

\section{Results}

Twenty-five patients (21 females and 4 males) with biochemically diagnosed hypercortisolism were evaluated by CT and MRI. The age range was $8-55$ years (average age 30 years). Three patients underwent an MR scan twice for recurrent symptoms after transsphenoidal pituitary microsurgery. MRI revealed adenomas in 16 patients (12 microadenomas and 4 macroadenomas). Nine patients had no abnormality either on CT or MRI. An analysis of the MR appearance of the adenomas $(n=16)$ showed that on pre-gadolinium scans $9(56 \%)$ of the adenomas were isointense, 7 (44\%) were hypointense and none was hyperintense to the native pituitary gland. On post-gadolinium studies, 15 (93.2\%) adenomas appeared hypointense (fig. 1) and only 1 (8\%) was isointense, and hence the adenomas were visualized better on pre-gadolinium images. Twelve (75\%) adenomas were delineated more clearly on post-gadolinium scans and $3(25 \%)$ equally well on pre- and post-gadolinium images. Only 1 adenoma was seen better on pre-contrast studies. 


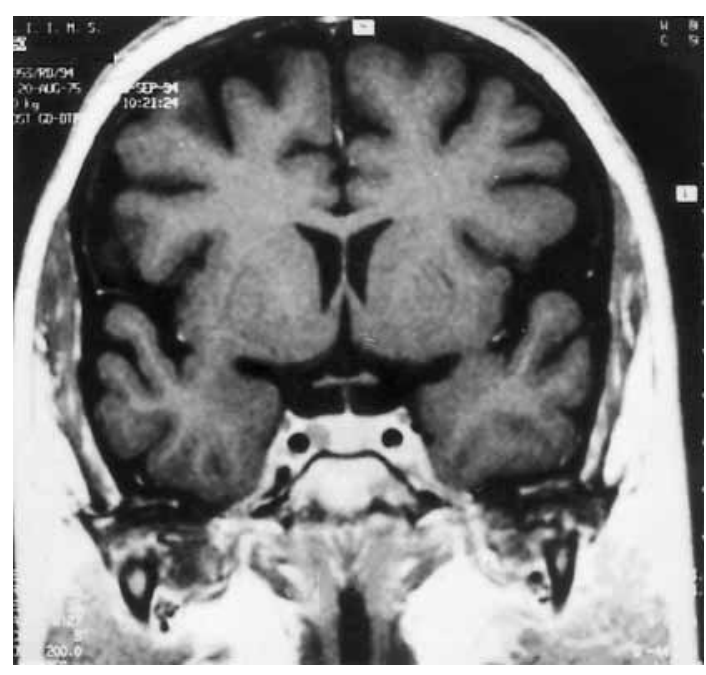

Fig. 1. Post-gadolinium, $T_{1}$-weighed $M R I$ in the coronal plane showing a round, well-defined, hypointense lesion in the right half of the pituitary gland suggestive of a microadenoma.

All pituitary glands were examined by serial sagittal and coronal scans. Nine (56\%) adenomas were identified much better on coronal images, and $6(37.5 \%)$ were visualized equally well on coronal and sagittal images. Only 1 $(6.2 \%)$ adenoma, which was located in the far anterior of the pituitary gland, was evaluated better on sagittal scans.

On MR examination, bulging of the superior surface of the pituitary was seen in all cases with underlying adenoma except 1 . A focal bulge was identified on the superior surface of $11(44 \%)$ pituitary glands, a diffuse bulge on 4 $(16 \%)$, while no abnormality of the superior surface was seen in 10 cases $(40 \%)$.

A deviation of the infundibulum away from the lesion was seen in 7 cases (44\%). In 9 cases (56\%), the infundibulum was midline and hence did not contribute to the localization of the tumour.

Suprasellar extension was seen in 3 cases $(18.7 \%)$ and sphenoid sinus extension was present in 1 case (6.2\%). Two cases revealed a breach of the sellar floor, 1 of them being postoperative.

Contrast-enhanced CT scans of the 5 sella scans $(25 \%)$, revealed intrasellar adenomas. Twenty scans were normal. Out of the 16 adenomas identified on MRI, only 5 were seen on the CT scans: 3 macroadenomas and 2 microadenomas. CT showed bulging superior surface in all, and deviation of stalk was detected in 2 cases.

All patients with pituitary adenoma imaged on MR underwent trans-sphenoidal microsurgery. Intra-operative bleeding precluded adequate exposure of the gland in 2 patients. These patients were subsequently subjected to bilateral adrenalectomy. Of the 9 patients with normal pituitary MR scans, the one with the enlarged pituitary underwent pituitary exploration and was subsequently proven by histopathology to be harbouring a microadenoma. Seven patients underwent bilateral adrenalectomy. One patient, who also had a breast carcinoma, died before surgery.

\section{Discussion}

Cushing's disease is caused by pituitary hypersecretion of ACTH. Pituitary adenomas are demonstrable in over $75 \%$ of the cases [ 2 , 3]. Abnormal hypothalamic pituitary function may be responsible for the hypersecretion of $\mathrm{ACTH}$ in the remaining patients $[4,5]$. Transsphenoidal resection of the adenoma is currently the preferred modality of treatment for patients with the disease $[2,3,6]$.

About $73-88 \%$ of the ACTH-secreting tumours are microadenomas $[2,7]$ the mean size being $4.5-6 \mathrm{~mm}[1,2,8]$. This small size accounts for the fact that it is difficult to detect the ACTH-secreting microadenomas despite pronounced clinical manifestations. The sensitivity of CT in detecting these mi- 
croadenomas varies from 17 [9] to 57\% [10]. Marcovitz et al. [7] reported a sensitivity of $63 \%$ and a specificity of $62.5 \%$ for ACTHsecreting adenomas with direct coronal CT. However, they reported a sensitivity of only $58 \%$ for microadenomas. Microadenomas other than those secreting ACTH are generally easier to detect on CT, as these tumours are larger at the time of presentation. Marcovitz et al. $[11,12]$ reported $81.2 \%$ sensitivity for detecting growth hormone microadenomas and a $91.9 \%$ sensitivity for prolactin microadenomas. Wu et al. [13] reported $100 \% \mathrm{MR}$ sensitivity in the detection of non-ACTHsecreting microadenomas. In comparison, contrast-enhanced CT detected $95 \%$ of the microadenomas in their series. These microadenomas were larger than $5 \mathrm{~mm}$ and hence easier to detect on CT.

MRI is the diagnostic modality of choice for detecting ACTH microadenomas. Kulkarni et al. [14] reported a sensitivity of $83 \%$ for MRI in detecting microadenomas at 1.5-tesla compared with $42 \%$ for CT. Dwyer et al. [15] reported $67 \%$ sensitivity on pre-gadolinium and $83 \%$ sensitivity on post-gadoliniumDTPA enhancement scan; CECT detected only $33 \%$ of these adenomas. Peck et al. [1] reported a sensitivity of $71 \%$ and a specificity of $87 \%$ in detecting ACTH-secreting microadenomas on a 1.5-tesla MR system. In our series, adenomas were detected in 16 out of 25 patients (64\%). Most of the adenomas were either isointense or hypointense on pregadolinium images, but on post-gadolinium studies, the majority (92\%) were hypointense. This is in consonance with findings reported earlier [13, 15]. Kucharcryk et al. [16] reported a predominance of hypointense lesions on $\mathrm{T}_{1}$-weighted images and a more variable appearance of adenomas on $\mathrm{T}_{2}$-weighted images. $T_{2}$-weighted images have long been known to provide little additional information and have since been abandoned [1]. In keeping with these recommendations, we have used only $T_{1}$-weighted images in the sagittal and coronal planes for evaluation of the pituitary gland. None of the adenomas were hyperintense on the $\mathrm{T}_{1}$-weighted images. Two microadenomas which were isointense on pre-gadolinium images were visualized only on post-gadolinium scans. In the series by Dwyer et al. [15], an additional 2 lesions were seen after gadolinium-DTPA enhancement. This has been attributed to early enhancement of the pituitary gland (maximum at 3 min after injection), as compared to the microadenoma which, as a result, is seen as hypointense lesion $[8,15]$.

Lesions were detected much more clearly in the coronal scans (56\%) and equally well $(38 \%)$ in the sagittal scans. Only in 1 case did the sagittal images delineate the adenoma more clearly. This emphasizes the superiority of coronal scans in displaying the two halves of the pituitary gland and any asymmetry of the signal intensity therein [1]. Moreover, sagittal sections tend to produce a partial-volume phenomenon with the intracavernous carotid artery.

The secondary signs of a pituitary adenoma are less sensitive than direct visualization of hypointensity within the pituitary gland. Among these, a focal or diffuse bulge in the superior surface of the pituitary gland was the most sensitive and seen in $60 \%$ of our cases. Infundibular displacement was less sensitive, and seen in 11 out of 25 cases. It has been reported to be of little value in localizing the tumour [10, 17]. Although Peck et al. [1] in their series of 16 patients reported stalk displacement in 12 cases, they accepted it only as a confirmatory sign and advocated a policy of discounting in the presence of focal hypointensity on the side ipsilateral to the deviational stalk. Sphenoid sinus extension was seen in only 1 case before operation and hence did not contribute to tumour localization. 
In conclusion, contrast-enhanced MRI is the preferred modality for the detection of ACTH-secreting adenomas which are difficult to visualize on $\mathrm{CT}$ scans due to their small size. A focal hypointensity is the commonest appearance on $\mathrm{T}_{1}$-weighted images, and, if identified on coronal scans, is the most sensitive diagnostic criterion. However, a negative MRI does not exclude the presence of a microadenoma.

\section{Acknowledgments}

We thank Ms. Sally Roy for her expert technical assistance and James D'Almeida for typing the manuscript.

\section{References}

1 Peck WW, Dillon WP, Norman D, Newton TH, Wilson CB: High-resolution MR imaging of pituitary microadenomas at $1.5 \mathrm{~T}$ : Experience with Cushing disease. AJR Am J Roentgenol 1989;152:145-151.

2 Boggan JE, Tyrrell JB, Wilson CB: Transsphenoidal microsurgical management of Cushing disease. Report of 100 cases. J Neurosurg 1983;59: 195-200.

3 Tyrrell JB, Brooks RM, Fitzgeralds PA, Cofoid PB, Forstam PH, Wilson CB: Cushing's disease - Selective trans-sphenoidal resection of pituitary microadenomas. N Engl J Med 1978;298:753-758.

4 Imura H: Ectopic hormone syndrome. Clin Endocrinol Metab 1980;9:235-360.

5 Feldman JM: Cushing's disease: A hypothalamic flush? N Engl J Med 1975;293:930-931.

6 Mampalam TJ, Tyrrell JB, Wilson $\mathrm{CB}$ : Transsphenoidal microsurgery for Cushing disease. A report of 216 cases. Ann Intern Med 1988;109: 487-493.
7 Marcovitz S, Wee C, Chan J, Hardy $\mathrm{J}$ : The diagnostic accuracy of preoperative CT scanning in the evaluation of pituitary ACTH-secreting adenomas. AJNR AM J Neuroradiol 1987;8:641-644.

8 Newton DR, Dillon WP, Norman D, Newton TH, Wilson CB: GdDTPA-enhanced MR imaging of pituitary adenomas. AJNR Am J Neuroradiol 1989;10:949-954.

9 Saris SC, Patronas NJ, Doppman JL, Loriaux DL, Cutler GB Jr, Nieman LK, Chrousos GP, Oldfield EH: Cushing syndrome: Pituitary CT scanning. Radiology 1987;162: 775-777.

10 Teasdale E, Teasdale G, Mohsen F, MacPherson P: High resolution computed tomography in pituitary microadenoma: Is seeing believing. Clin Radiol 1986;37:227-232.

11 Marcovitz S, Wee R, Chan S, Hardy $\mathrm{J}$ : Diagnostic accuracy of preoperative CT scanning of pituitary somatotrophy adenomas. AJNR Am J Neuroradiol 1988;9:19-22.

12 Marcovitz S, Wee R, Chan J, Hardy $\mathrm{J}$ : Diagnostic accuracy of preoperative scanning of pituitary prolactinomas. AJNR Am J Neuroradiol 1988;9:13-17.
$13 \mathrm{Wu}$ W, Thomas KA: Pituitary microadenomas: MR appearance and correlation with CT. Acta Radiol 1995;36:529-535.

14 Kulkarni M, Lee KF, McArdle C, Yeakley JW, Haar FL: 1.5-T MR imaging of pituitary microadenomas: Technical considerations and CT correlation. AJNR Am J Neuroradiol 1988;9:5-11.

15 Dwyer AJ, Frank JA, Doppman JL, Oldfield EH, Hickey AM, Cutler GB, Loriaux DL, Schiable TF: Pituitary adenoma in patients with Cushing disease: Initial experience with Gd-DTPA-enhanced MR imaging. Radiology 1987;163:421426.

16 Kucharcryk W, Davis DO, Kelly WM, Sze G, Norman D, Newton TH: Pituitary adenomas: High resolution MR imaging at $1.5 \mathrm{~T}$. Radiology 1986;161:761-765.

17 Raji MR, Kishore PRS, Becker DP: Pituitary microadenoma: A radiological surgical correlative study. Radiology 1981;139:95-99. 